\title{
Cohesion and Coordination Effects on Transition Metal Surface Energies
}

\author{
Judit Ruvireta, Lorena Vega, and Francesc Viñes* \\ Departament de Ciència de Materials i Química Física \& Institut de Química Teòrica i \\ Computacional (IQTCUB), Universitat de Barcelona, c/ Martí i Franquès 1, 08028 Barcelona, \\ Spain. \\ *Corresponding Author: francesc.vines@ub.edu
}

\begin{abstract}
Here we explore the accuracy of Stefan equation and broken-bond model semiempirical approaches to obtain surface energies on transition metals. Cohesive factors are accounted either via the vaporization enthalpies, as proposed in Stefan equation, or via cohesive energies, as employed in the broken-bond model. Coordination effects are considered including the saturation degree, as suggested in Stefan equation, employing Coordination Numbers $(\mathrm{CN})$, or as the ratio of broken bonds, according to bond-cutting model, considering as well the square root dependency of the bond strength on the $\mathrm{CN}$. Further, generalized coordination numbers $\overline{C N}$ are contemplated as well, exploring a total number of 12 semiempirical formulations on the three most densely packed surfaces of $3 d, 4 d$, and $5 d$ Transition Metals (TMs) displaying face-centered cubic (fcc), body-centered cubic (bcc), or hexagonal close-packed (hcp) crystallographic structures. Estimates are compared to available experimental surface energies obtained extrapolated to zero temperature. Results reveal that Stefan formula cohesive and coordination dependencies are only qualitative suited, but unadvised for quantitative discussion, as surface energies are highly overestimated, favoring in addition the stability of under-coordinated surfaces. Broken-bond cohesion and coordination dependencies are a suited basis for quantitative comparison, where square-root dependencies on $\mathrm{CN}$ to account for bond weakening are sensibly worse. An analysis using Wulff shaped averaged surface energies suggests the employment of broken-bond model using $\mathrm{CN}$ to gain surface energies for TMs, likely applicable to other metals.
\end{abstract}

Keywords: Surface Energy $\cdot$ Transition Metals $\cdot$ Broken-Bond Model $\cdot$ Stefan Equation - Surface Tension · Wulff Construction 


\section{Introduction}

Surfaces are the main defect on an otherwise infinite material. In fact crystalline materials periodically repeat their bulk structure along the three-dimensional space up to the surface terminations. Material nanoparticles, micrograins, up to macroscopic single crystals expose certain preferential surface endings, which in turn outline the particle shape. Infinite plane endings exist within a crystal, yet groups of them are equivalent due to the materials bulk intrinsic crystal group symmetry. The natural observed trend is that materials expose those terminations or surfaces families that are more stable, typically being the most close-packed ones. The rationalization is simple; those terminations where surface atoms are more saturated lack less bonds, and so, the fewer bonds are missed, the more stable the surface is.

The stability of a given surface termination is quantified by the so-called surface energy, $\gamma$, also known as surface tension, given per exposed surface area - typically in $\mathrm{J}$ $\mathrm{m}^{-2}$ or $\mathrm{N} \mathrm{m}^{-1}$ units. Ideally, when a bulk is truncated into two surface endings, the cleavage energy equals to both surface energies added up. Surface energies include immediate post-cleavage effects such as surface relaxation or atomic reconstructions. These processes are considered secondary though, and the materials bulk cohesive strength is actually normally regarded as the main property sizing the surface energy. Again the concept is straightforward; the stronger the bonds in the material are, the most costly to create a surface is, and, therefore, the higher the surface energy.

The surface energy is the main energetic descriptor of a crystal termination, and many physicochemical surface properties hang upon it. For instance, Wulff construction procedure is used as a top-down approach to ascertain the crystal equilibrium shape from independent surface energies [1]. The moiety shape can affect the overall electronic structure of material nanoparticles, and even alter their magnetic solution $[2,3]$. Furthermore, the presence of certain surface terminations can induce particular moiety aggregations, such as metal nanowires from isolated nanoparticles [4]. The different surface terminations differ in their electronic structures, and so surface properties depend on them, e.g. the work function, of vital importance in processes where electrons are ejected from the material, like in electrochemical processes, or surface science techniques like the X-ray photoemission spectroscopy and scanning tunneling microscopy.

On top of that one has to regard that surfaces are the main region where materials interact with media, and so, of pivotal importance in cutting-edge surface- 
driven processes where nowadays research focus onto, e.g. chemical resolution of enantiomers [5], $\mathrm{CO}_{2} / \mathrm{CH}_{4}$ gas separation [6], patterning of two-dimensional organic frameworks [7], electron/hole separation in photocatalysis [8], and heterogeneous catalysis [9], to cite a few examples of technological relevance. Moreover, a given structural shape in turn expresses other lower dimensionality defects such as edges and corners, which may also feature, due to their lower atomic coordination, a markedly different chemical activity [10-12]. Shape, size, and surface tension are determining factors of transition metal nanoparticles reactivity [13-15], where surface energies have been claimed as a main indicator of the overall metal surface activity [16], and because of this posed as a descriptor of its catalytic activity [17]. As it happens, other coordination [18] and electronic [19] descriptors are intimately linked to a particular surface termination.

The experimental measurement of a solid metal surface energy is challenging: On one hand it is important to measure a system without impurities, which otherwise could substantially affect the determined $\gamma$. On the other hand, the employed techniques typically require having the metal (nearly) molten. Different procedures can be employed, such as sessile and pendent drops, drop weight, capillary rise, and maximum bubble or drop pressures, for whose detailed description we refer to the literature [20]. In all cases, the $\gamma$ drops with the temperature, but only ranging $0.1-0.4 \mathrm{mN} \mathrm{m}^{-1} \mathrm{~K}^{-1}$, this is, the effect of the temperature only becomes significant at very large temperatures above $1000 \mathrm{~K}$ [21]. In any case, from the linear dependence of $\gamma$ with respect the temperature $\mathrm{T}$ on can gain extrapolated surface energy values at $0 \mathrm{~K}$, with multiple acquired values over the last decades, ensuring finite values with an overall associated error of $\pm 2 \%[21]$.

These experimentally extrapolated $\gamma$ values arise from (nearly) molten metal situations, with a highly dynamical admixing of particular surface endings, and because of that, difficult to assign to a particular crystallographic ending. This lack of atomistic knowledge on surface energies from the experimental point of view can be mended from the computational chemistry one, where density functional theory calculations on slab models are commonly used to mimic specific surface endings under study, and to obtain estimates of their surface energies. These estimates allow comparing the experimental surface energies with those obtained on slab models of most stable surfaces [22], as they are presumably those most exposed on a nanocrystallite according 
to Wulff construction [1,23], or even to a combination of surface endings of low Miller indices surfaces, profiting from a Wulff constructed shape [24].

Despite of the benefits on acquiring surface energy values from DFT calculations, some questions are to date under debate. Focusing only on transition metals as a large representative subset of metallic systems, one may wonder whether the employment of a given exchange-correlation (xc) functional may bias the surface energy estimates; in this regard, it seems clear that xc functionals within the generalized gradient approximation are best suited in describing metal bulks [22,25], although the question mark is still present when addressing metal surfaces. Furthermore, the modeling and optimization of surface slabs is computational demanding, and thorough studies are limited nowadays to maximum miller indices of two [24], despite surfaces with higher Miller indices can be highly stable and so present in any system, or stabilized in case of their technological importance; see e.g. $\mathrm{Cu}(321)$ surface utilization in the catalysis of the water gas shift [26], or $\mathrm{Cu}(3117)$ chiral metal surfaces used for enantioselective chemical separation [27], to mention a couple of cases.

In that sense, the usage of semiempirical equations to estimate the surface energies of a particular surface ending becomes quite appealing, given their easiness and rapid utilization, more if, in addition, they are able to deliver surface energies with a high degree of accuracy. Historically, one has to remark the Stefan equation [28], in which the surface energy can be obtained as;

$$
\gamma=\frac{\Delta H_{v a p}^{o} \rho^{2} / 3}{M^{2} / 3 N_{A}^{2} / 3} \frac{C N_{S}}{C N_{b}}
$$

where $\Delta H_{v a p}^{o}$ is the material vaporization at standard conditions of pressure and temperature $-10^{5} \mathrm{~Pa}$ and $273.15 \mathrm{~K}-, \rho$ is the material density, $M$ the molar mass, and $N_{A}$ the Avogadro constant. Aside from these variables, the surface energy depends on the relation in between the Coordination Number $(\mathrm{CN})$ of the surface $\left(\mathrm{CN}_{\mathrm{s}}\right)$ with respect that of the material bulk $\left(\mathrm{CN}_{\mathrm{b}}\right)$. The Stefan equation can be then decomposed into two clearly differentiated terms; the $\mathrm{CN}_{\mathrm{s}} / \mathrm{CN}_{\mathrm{b}}$, which quantifies the degree of saturation of surface atoms with respect bulk conditions, and the rest of the equation, which is a weighted value of $\Delta H_{v a p}^{o}$, accounting for the materials atomic cohesion. Hence, these terms account for the above-stated cohesion and coordination dependences, and stem from the experimental observation of dependence of $\gamma$ with respect $\Delta H_{v a p}^{o}$ [20], and $\mathrm{CN}_{\mathrm{S}} / \mathrm{CN}_{\mathrm{b}}$ terms [29]. 
The other extendedly used empirical adjustment is that of the bond-broken model, also known as bond-cutting model [30]. There the surface energy $\gamma$ is expressed as

$$
\gamma=\frac{C N_{b}-C N_{s}}{C N_{b}} E_{c o h}
$$

where $E_{c o h}$ is the material cohesive energy, which naturally accounts for the material cohesion dependence. At variance with Stefan equation, the saturation proportionality term depends on $\mathrm{CN}_{\mathrm{b}}-\mathrm{CN}_{\mathrm{s}}$, this is, the number of broken bonds when creating the surface. Criticism arose on the broken-bond model in the sense that ignores the variation of bond strengths with respect $\mathrm{CN}$ [31], such as in the above commented surface relaxation processes, and lower dimensionality defects. Tight-binding theory showed a square root dependency on $\mathrm{CN}$ for covalent bonds [32], and so the broken bond equation could be reformulated as

$$
\gamma=\frac{\sqrt{C N_{b}}-\sqrt{C N_{s}}}{\sqrt{C N_{b}}} E_{c o h}
$$

where its usage on transition metals has been advised, claiming an agreement compared to $a b$ initio estimates being improved by $20-50 \%$ [33-35], although the explicit agreement or improvement with respect experimental values was not addressed.

At this point, it is clear that many questions remain open when using these empirical equations: What cohesion term is better suited, standard vaporization enthalpies or cohesive energies? Moreover, is surface saturation better treated in terms of coordination, or as broken bonds? Would the above commented square root dependency apply when comparing to experimentally determined surface energies? On top of that, recently generalized coordination numbers $(\overline{C N})$ have been suggested and employed as better geometric descriptors of the transition metals surface activity compared to $\mathrm{CN}$ [36,37], allowing distinguishing different similarly packed surfaces, by considering the saturation of subsurface and vicinal lower-dimension sites. In this sense, would $\overline{C N}$ be a better coordination parameter to quantify the surface stability in terms of $\gamma$ ? To solve these questions, we here present a profound analysis on the parameterization and dependences employed for the cohesion and coordination effects, by comparing surface energies obtained based on Stefan equation and broken-bond models, compared to precise values of surface energies considering 26 transition metals as a broad and representative study set. 


\section{Computational Details}

Different empirical equations have been tested, either based on Stefan equation employing standard vaporization energies as cohesion descriptor, or based on the broken-bond model, and, therefore utilizing cohesive energies for that purpose. The list of experimental standard vaporization energies, densities, and cohesive energies is found in the Supplementary Information. These cohesion terms are combined with the above stated

$$
\text { (1) } \frac{C N_{S}}{C N_{b}}, \text { (2) } \frac{C N_{b}-C N_{S}}{C N_{b}} \text {, or (3) } \frac{\sqrt{C N_{b}}-\sqrt{C N_{S}}}{\sqrt{C N_{b}}}
$$

cohesion terms, either employing standard coordination numbers, $C N$, or generalized coordination numbers, $\overline{C N}$. This implies the screening of 12 possible empirical relationships. The selected 26 Transition Metals (TMs) are those which feature a face centered cubic (fcc) crystallographic arrangement ( $\mathrm{Ni}, \mathrm{Cu}, \mathrm{Rh}, \mathrm{Pd}, \mathrm{Ag}, \mathrm{Ir}, \mathrm{Pt}$, and $\mathrm{Au}$ ), a body centered cubic (bcc) one (V, Cr, Fe, Nb, Mo, Ta, and W), or an hexagonal closed packed (hcp) crystal structure (Sc, Ti, Co, Zn, Y, Zr, Ru, Cd, Hf, Re, and Os), whose zero temperature surface energies, $\gamma$, are known, extrapolated from $\gamma$ versus T plots [21], and here listed in the Supplementary Information. The bulk $\mathrm{CN}_{\mathrm{b}}$ - and also $\overline{C N}_{b}-$ are 12 for fcc and hcp transition metals, and 8 for bcc metals, see Table 1.

Concerning $\mathrm{CN}_{\mathrm{s}}$ - and also $\overline{C N}_{s}$-, three different surfaces have been contemplated for each family of metals, considering a maximum Miller index order of one. These are the (001), (011), and (111) surfaces of fcc anc bcc TMs, and (0001), (1010), and $(11 \overline{2} 0)$ surfaces for hcp TMs, which a priori include always the most stable exposed surface, that with highest packing; the (011), (111), and (0001) surfaces for bcc, fcc, and hcp TMs, respectively. Model surfaces allow one for acquiring surface CNs and $\overline{C N}_{S}$ values, following a recipe previously described [37]. The obtained surface energy values are used to build Wulff construction shapes [23], from which ratios of surface exposure can be used to obtain surface-weighted mean nanoparticle surface energies.

\section{Results and Discussion}

To being with, the most stable obtained surface energy with any of the examined 12 empirical equations for each TM under study has been compared to its experimentally determined surface energy. This comparison is extendedly used in research [16], and the reason for that is the assumption that, in any metal system, the most exposed surface is that most stable, a rule-of-a-thumb not exempt of criticism and exceptions, specially 
when Wulff constructed shapes limit the extent of such an argument [23], although, regardless of that, one can consider such approximation as a good initial guess, just succinctly modified (increased surface energies) when considering other less stable surfaces.

All that said, the comparison serves as a first screening, and clearly shows that the Stefan equation, as originally proposed (eq. 1), is exceedingly overestimating the surface energies, see Figure 1. The $\mathrm{CN}_{\mathrm{s}} / \mathrm{CN}_{\mathrm{b}}$ coordination relation implies that surface energies are lower for those terminations with less surface coordination, contrary to the above-commented conceptualization of higher stability associated to a larger coordination. This relation then implies a much higher surface energy for denselypacked terminations, which explains the overestimation when plotting the most densely packed (011), (111), and (0001) surfaces for bcc, fcc, and hcp TMs, respectively. However, the differences in surface energy values because of such coordination dependence range 1-3 $\mathrm{J} \mathrm{m}^{-2}$, and so, just partly account for the full overestimation, where the rest stems out from the energetic dependency on $\Delta H_{v a p}^{o}$.

To further support that, notice that the overestimation is greatly improved when using the original broken-bond model coordination relation (eq. 2). There the most stable surfaces are the employed highly-packed, and so, usage of other surfaces would only increase the discrepancy. Furthermore, the coordination effect based on missing bonds seems more suited to reality, although still overestimating. Indeed, the squareroot dependency of bond strength on coordination (eq. 3) further counteracts this overestimation, with values comparable to the experimental ones. One has to remark that, regardless of the explored coordination proportionality, the cohesion dependence is always followed, and so, despite the overestimation(s), one can use such dependences to qualitatively claim a higher surface energy of one material to another, by simply comparing vaporization energies, a fact of possible usage in treating bimetallics or alloys of different compositions. Concerning the employment of generalized coordination numbers, $\overline{C N}$, the improvement is clear on Stefan equation, as the $\overline{C N}_{S} / \overline{C N}_{b}$ decreases, but slightly detrimental for broken-bond based relationships. Therefore its usage would only be advised for qualitative comparative purposes.

The cohesion dependence on $\mathrm{E}_{\mathrm{coh}}$ is tackled in Figure 2, and, compared to $\Delta H_{\text {vap }}^{o}$ based results in Figure 1, one immediately sees that cohesive energies largely bridge the gap of the above-commented overestimation, with obtained values much closer to the 
experimentally determined ones. Because of this, overall, the broken-bond model is better suited than Stefan equation in accurately determining TM surface energies, and likely, other metals and materials. In any case the mutual relative surface energies among metals are correctly described at any explored combination of terms, given the linear dependence of vaporization enthalpy with respect cohesive energy. That said, the $\mathrm{CN}_{\mathrm{s}} / \mathrm{CN}_{\mathrm{b}}$ or $\overline{C N}_{s} / \overline{C N}_{b}$ relationships are unadvised, as they favor the less saturated surfaces, as above commented. The question mark here is whether the direct (generalized) coordination number or the square root dependency is better. An ideal agreement with the experimental values should deliver linear dependency with both regression coefficient and slope of unity, with an intercept at zero. Taking this into account, the linear dependencies, as provided in the Supplementary Information, render the broken-bond model based equation with direct (generalized) coordination numbers as best in estimating surface energies, with intercepts of -0.04 and $-0.05 \mathrm{~J} \mathrm{~m}^{-2}$ - thus in the range of experimental accuracy of $\pm 2 \%[21]$ - slopes of 0.81 and 1.22 , when using $\mathrm{CN}$ or $\overline{C N}$, respectively, and regression coefficients $R$ in both cases of 0.86 . Notice that the claimed improvement when using root-square dependencies does not apply for TMs, and so, the original broken-bond equation (eq. 2) is better suited, likely extendable to other metals.

At this point one may wonder whether such comparison of the experimental values to the most stable (packed) surface is well sustained, and whether neglecting of the other less stable surfaces implies deviations on such linear dependencies. To further investigate this, we here constructed Wulff shapes $[1,23]$ with the above commented estimates of surface energies, either based on $\mathrm{CN}$ or $\overline{C N}$ coordination, for fcc, bcc, and hcp metals, see Figure 3. Notice how the usage of generalized coordination numbers slightly favors the appearance of less stable (001) surfaces on fcc and bcc TMs, although no effect is visible in hcp transition metals. In the case of fcc metals, the Wulff shape is a truncated octahedron composed of (111) facets with small truncated (001) ones. In the case of bcc, the Wulff shape is a dodecahedron exposing (011) facets, although some truncation could be visible showing (001) facets. In the case of hcp TMs, the equilibrium Wulff shape is an hexagonal prism featuring with hexagons being the (0001) surfaces, with (1010) edges.

Table 2 contains the surface ratio as extracted from Wulff shapes in Figure 3, which has been used to leverage the surface energies of the above-commented broken- 
bond equations using either $\mathrm{CN}$ or $\overline{C N}$ coordination numbers. The obtained mean surface energies are then compared to the experimental values in Figure 4. Notice how the inclusion of less packed and so less stable surfaces slightly increases the mean surface energies. While this effect is detrimental when using generalized coordination numbers in the broken-bond model, where former values where slightly overestimated, it is beneficial when using regular coordination $\mathrm{CN}$ numbers, as they were formerly slightly underestimated, thus approaching the estimated values to the experimental reported ones. This is reflected in the linear regressions, where linear fitting using $\overline{C N}$ delivers a worse $R$ coefficient of 0.80 , a worse intercept of $0.12 \mathrm{~J} \mathrm{~m}^{-2}$, and only a slight improvement of the slope of 1.15 . When using $\mathrm{CN}$, the $R$ is slightly worsened to 0.82 , whereas both intercept - almost matching value of $-0.01 \mathrm{~J} \mathrm{~m}^{-2}$ - and slope — value of 0.87- are improved. The average associated errors on the cohesive energy estimates are of $0.1 \%$ and $0.1 \%$ when using $\mathrm{CN}$ and $\overline{C N}$, respectively, well below the experimental mean error bars of $2 \%$ [21].

All in all, present results comparing Wulff shape averaged surface energies to zero temperature extrapolated surface energies highlight the employment of the original formulation of the broken-bond model as a better suited empirical formula to estimate surface energies of transition metals, although results are probably extendable to other metals. The cohesion dependence on vaporization enthalpies and surface saturation as employed in Stefan formulation are unadvised for quantitative determinations, and so should be employed only for qualitative discussion. The employment of generalized coordination numbers is not critical, and so could be used for quantitative determinations, although regular coordination numbers are here found to be slightly better suited.

\section{Conclusions}

Here we have explored the validity and accuracy of two main semiempirical approaches to rapidly obtain estimates of surface energies. These are the Stefan equation and the broken-bond model. Cohesive factors are contemplated either via the vaporization enthalpies as proposed in the Stefan equation, or via the cohesive energies, as employed in the broken-bond model. Coordination effects are accounted including the saturation degree as employed in Stefan equation using $\mathrm{CN}$, or as the ratio of broken bonds following the broken-bond model, including the square root dependency of the bond strength on the $\mathrm{CN}$, as posteriorly proposed to deliver much accurate estimates. Further 
than that, the usage of $\overline{C N}$ instead of $\mathrm{CN}$ has been contemplated, accounting for a total number of 12 explored empirical formulations of surface energies, which have been tested on the three most densely packed and presumably most stable surfaces of $3 d, 4 d$, and $5 d$ TMs displaying fcc, bcc, or hep crystallographic structures.

The obtained results on the most densely packed surface compared to experimentally available zero temperature extrapolated surface energies reveal that Stefan formula cohesive and coordination dependencies are suited for a qualitative analysis, but they are unadvised for quantitative discussion, as the obtained surface energies are highly overestimated, favoring the stability of under-coordinated surfaces. The same comparison grants the broken-bond cohesion dependency on cohesive energies a suited basis for a quantitative discussion, where the original coordination dependency based on missing bonds yields the best agreement with experiments, at variance with square-root dependencies, which are sensibly worse. A further analysis comparing Wulff shaped averaged surface energies with experiments further suggests the employment of the broken-bond model as a quantitatively accurate semiempirical method to gain surface energies for TMs, likely applicable to other metals.

Acknowledgements: This work has been supported by Spanish Ministry of Economy and Competitiveness (MINECO) CTQ2015-64618-R, and partly by Generalitat de Catalunya grants 2014SGR97 and XRQTC. F.V. thanks MINECO for a postdoctoral Ramón y Cajal (RyC) research contract (RYC-2012-10129). 
Table 1. Bulk and surface coordination numbers, $\mathrm{CN}_{\mathrm{b}}$ and $\mathrm{CN}_{\mathrm{s}}$, respectively, and generalized bulk and surface coordination numbers, $\overline{C N}_{b}$ and $\overline{C N}_{s}$, respectively, for the different surfaces under study of bcc, fcc, and hcp TMs.

\begin{tabular}{cccccc}
\hline Crystal & Surface & $\mathrm{CN}_{\mathrm{b}}$ & $\mathrm{CN}_{\mathrm{s}}$ & $\overline{C N}_{b}$ & $\overline{C N}_{s}$ \\
\hline \hline bcc & Bulk & 8 & \multicolumn{3}{c}{8} \\
& $(001)$ & & 4 & & 4.00 \\
& $(011)$ & & 6 & & 5.00 \\
& $(111)$ & & 4 & & 3.63 \\
fcc & Bulk & 12 & & 12 & \\
& $(001)$ & & 8 & & 6.67 \\
& $(011)$ & & 7 & & 5.83 \\
& $(111)$ & & 9 & & 7.50 \\
hcp & Bulk & 12 & & 12 & \\
& $(0001)$ & & 9 & & 7.50 \\
& $(10 \overline{1} 0)$ & & 8 & & 6.67 \\
& $(11 \overline{2} 0)$ & & 7 & & 5.83 \\
\hline
\end{tabular}


Table 2. Ratio of exposed surfaces as following the equilibrium Wulff shapes for fcc, bcc, and hcp TMs, see Figure 3.

\begin{tabular}{cccc}
\hline Wulff Crystal & Surface & CN & $\overline{C N}$ \\
\hline \hline bcc & $(001)$ & 0 & 0.010 \\
& $(011)$ & 1 & 0.990 \\
& $(111)$ & 0 & 0 \\
fcc & $(001)$ & 0.098 & 0.202 \\
& $(011)$ & 0 & \\
& $(111)$ & 0.902 & 0.798 \\
hcp & $(0001)$ & 0.333 & 0.333 \\
& $(10 \overline{1} 0)$ & 0.667 & 0.667 \\
& $(11 \overline{2} 0)$ & 0 & 0 \\
\hline
\end{tabular}


Figure 1. Stefan equation surface energies, $\gamma_{\text {theo, }}$, either using coordination numbers $(\mathrm{CN}$ - top panel) or generalized coordination numbers ( $\overline{C N}$ - bottom panel), employing the different coordination dependences as outlined in eq. 4, with respect the experimental values extrapolated to zero temperature [21], $\gamma_{\exp }$.

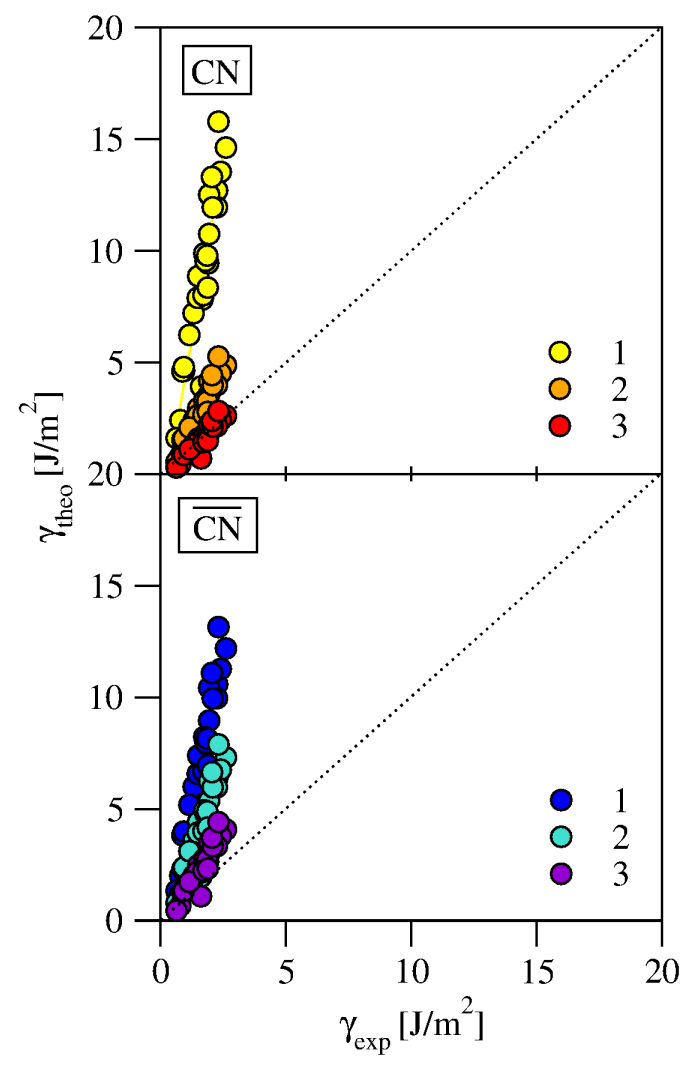


Figure 2. Broken-bond model equation surface energies, $\gamma_{\text {theo }}$, either using coordination numbers (CN - top panel) or generalized coordination numbers ( $\overline{C N}$ - bottom panel), employing the different coordination dependences as outlined in eq. 4 , with respect the experimental values extrapolated to zero temperature [21], $\gamma_{\exp }$.

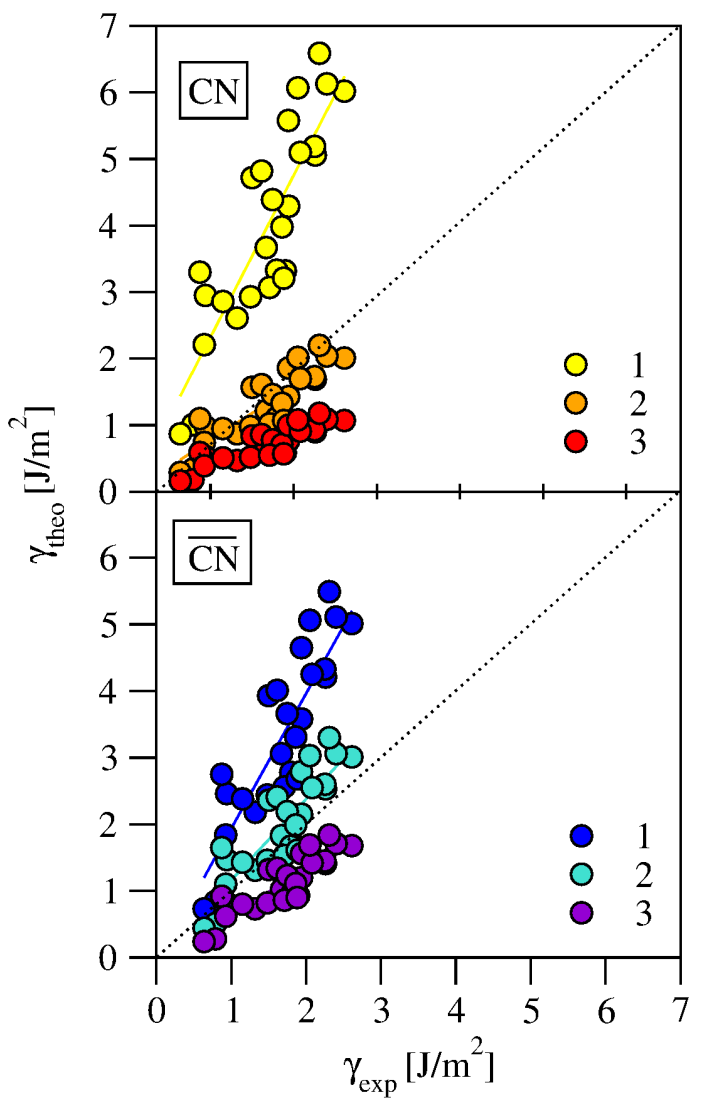


Figure 3. Wulff constructed shapes for fcc, bcc, and hcp metals, using the surface energies from the broken-bond model formula (eq. 2), either using the coordination numbers $(\mathrm{CN})$ or generalized coordination numbers $(\overline{C N})$.
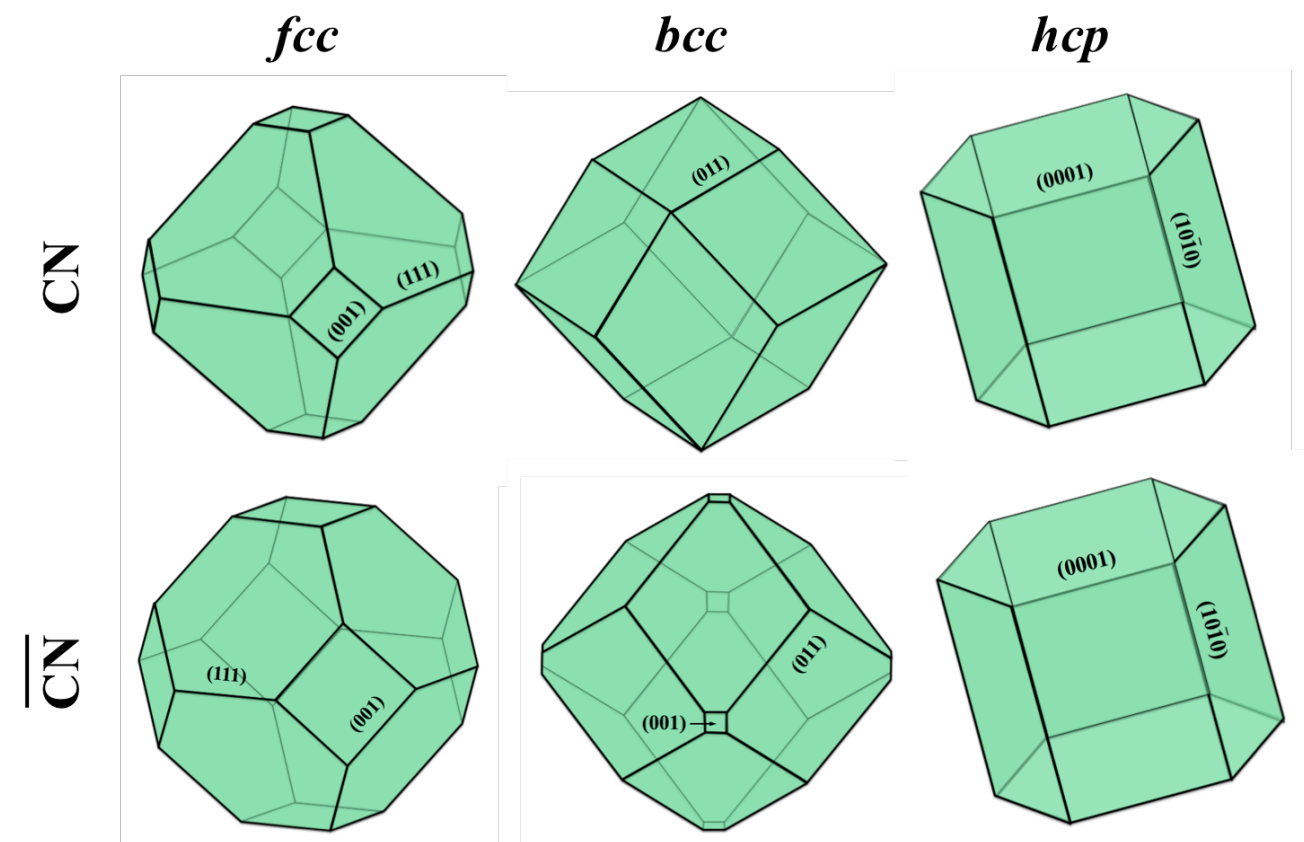
Figure 4. Surface energies obtained from the broken-bond model formula (eq. 2), either using the coordination numbers $(\mathrm{CN}-$ in green) or generalized coordination numbers ( $\overline{C N}$ - in blue), using the most stable surface energy (light colors) or surface averaged according to the Wulff constructed shapes depicted in Figure 3 (dark colors).

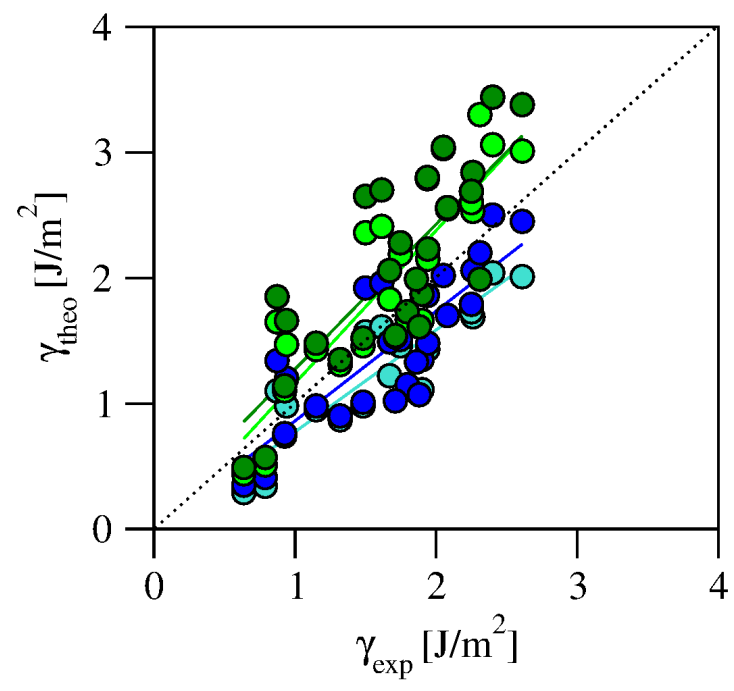




\section{References}

[1] G. Wulff, Z. Kristallogr. Mineral. 34 (1901) 449.

[2] F. Viñes, F. Illas, K.M. Neyman, J. Phys. Chem. A 112 (2008) 8911.

[3] J.L.C. Fajín, A. Bruix, M.N.D.S. Cordeiro, J.R.B. Gomes, F. Illas, J. Chem. Phys. 137 (2012) 034701.

[4] F. Viñes, F. Illas, K.M. Neyman, Angew. Chem. Int. Ed. 46 (2007) 7094.

[5] Y. Yun, A.J. Gellman, Angew. Chem. Int. Ed. 52 (2013) 3394.

[6] M.A.O. Lourenço, C. Siquet, M. Sardo, L. Mafra, J. Pires, M. Jorge, M.L. Pinto, P. Ferreira, J.R.B. Gomes, J. Phys. Chem. C 120 (2016) 3863.

[7] M. Pivetta, G.E. Pacchioni, E. Fernandes, H. Brune, J. Chem. Phys. 142 (2015) 101928

[8] A. Iglesias-Juez, F. Viñes, O. Lamiel-García, M. Fernández-García, F. Illas, J. Mater. Chem. A 3 (2015) 8782.

[9] C. Michel, F. Auneau, F. Delbecq, P. Sautet, ACS Catal. 1 (2011) 1430.

[10] J.L.C. Fajín, A. Bruix, M.N.D.S. Cordeiro, J.R.B. Gomes, F. Illas, J. Chem. Phys. 137 (2012) 034701.

[11] S.M. Kozlov, K.M. Neyman, J. Catal. 337 (2016) 111.

[12] F. Viñes, Y. Lykhach, T. Staudt, M.P.A. Lorenz, C. Papp, H.-P. Steinrück, J. Libuda, K.M. Neyman, A. Görling, Chem. Eur. J. 16 (2010) 6530.

[13] S. Mostafa, F. Behafarid, J.R. Croy, L.K. Ono, L. Li, J.C. Yang, A.I. Frenkel, B. Roldan Cuenya, J. Am. Chem. Soc. 132 (2010) 15714.

[14] E. Schmidt, A. Vargas, T. Mallat, A. Baiker, J. Am. Chem. Soc. 131 (2009) 12358.

[15] L. Li, F. Abild-Pedersen, J. Greeley, J.K. Nørskov, J. Phys. Chem. Lett. 6 (2015) 3797.

[16] P. Janthon, S.M. Kozlov, F. Viñes, J. Limtrakul, F. Illas, J. Chem. Theory Comput. 9 (2013) 1631. 
[17] H.L. Zhuang, A.J. Tkalych, E.A. Carter, J. Phys. Chem. C 120 (2016) 23698.

[18] F. Calle-Vallejo, D. Loffreda, M.T.M. Koper, P. Sautet, Nat. Chem. 7 (2015) 403.

[19] J.K. Nørskov, F. Abild-Pedersen, F. Studt, T. Bligaard, Proc. Natl. Acad. Sci. 108 (2011) 937.

[20] B.J. Keene, Int. Mater. Rev. 38 (1993) 157.

[21] K.C. Mills, Y.C. Su, Int. Mater. Rev. 51 (2006) 329.

[22] P. Janthon, S.M. Kozlov, F. Viñes, J. Limtrakul, F. Illas, J. Chem. Theo. Comput. 9 (2013) 1631.

[23] F. Viñes, J.R.B. Gomes, F. Illas, Chem. Soc. Rev. 43 (2014) 4922.

[24] R. Tran, Z. Xu, B. Radhakrishnan, D. Winston, W. Sun, K.A. Persson, S.P. Ong, Sci. Data. 3 (2016) 160080.

[25] P. Janthon, S. Luo, S.M. Kozlov, F. Viñes, J. Limtrakul, D.G. Truhlar, F. Illas, J. Chem. Theo. Comput. 10 (2014) 3832.

[26] H. Prats, P. Gamallo, F. Illas, R. Sayós, J. Catal. 342 (2016) 75.

[27] Y. Yun, A.J. Gellman, Angew. Chem. Int. Ed. 52 (2013) 3394.

[28] K.S. Birdi, Surface and Colloid Chemistry, Principles and Applications 2010, CRC Press

[29] A.A. Strechan, G.J. Kabo, Y.U. Paulechka, Fluid Phase Equilib. 250 (2006) 125.

[30] D. McLachlan, Acta Met. 5 (1975) 111.

[31] J. Pauling, J. Am. Chem. Soc. 53 (1931) 1367.

[32] V. Heine, J. Hafner, Many-Atom Interactions in Solids, Ed. R. Nieminen, M.J. Puska and M.J. Manninen 1990, Springer, Berlin, pp. 12.

[33] I.J. Robertson, M.C. Payne, V. Heine, Euro Phys. Lett. 15 (1991) 301.

[34] C. Hausleitner, J. Hafner, Phys. Rev. B 45 (1992) 115.

[35] A. Eichler, J. Hafner, J. Furthmüller, G. Kresse, Surf. Sci. 346 (1996) 300. 
[36] F. Calle-Vallejo, J. Tymoczko, V. Colic, Q.H. Vu, M.D. Pohl, K. Morgenstern, D. Loffreda, P. Sautet, W. Schuhmann, A.S. Bandarenka, Science 350 (2015) 185.

[37] F. Calle-Vallejo, J.I. Martínez, J.M. García-Lastra, P. Sautet, D. Loffreda, Angew. Chem. Int. Ed. 53 (2014) 1. 